\title{
Buchbesprechungen
}

Arne Duncker, Gleichbeit und Ungleichbeit in der Ehe. Persönliche Stellung von Mann und Frau im Recht der ebelichen Lebensgemeinschaft 1700-1914. Köln/Weimar/Wien (Böhlan Verlag, Rechtsgeschichte und Geschlechterforschung, Band 1) 2003, 1189 S., 94,-€.

Die hier vorgestellte Monographie mit dem schönen Titel »Gleichheit und Ungleichheit in der Ehe "ist als Bd. 1 einer neuen Reihe des Böhlau Verlages zur "Rechtsgeschichte und Geschlechterforschung « erschienen. Das Thema ist spannend und aktuell, denn die Vorstellungen und Leitbilder von Ehe, Familie und Paarbeziehungen sind in einem grundlegenden Wandel begriffen. Neue, verbindliche Leitbilder fehlen, und die daraus resultierende Unsicherheit betrifft Männer und Frauen in existentieller Weise. Selbst zeitgenössische Ehedefinitionen, etwa BVerfGE 10, 66: »Ehe ist auch für das Grundgesetz die Vereinigung eines Mannes und einer Frau zu einer grundsätzlich unauflöslichen Lebensgemeinschaft.«, erscheinen heute überholt, denn die lebenslange Gemeinschaft ist in der Realität kein vorherrschendes Merkmal gegenwärtiger Ehen mehr, und selbst das Merkmal Lebensgemeinschaft von Mann und Frau hat angesichts der zunehmenden Akzeptanz gleichgeschlechtlicher Lebensgemeinschaften in den EU-Staaten seine Verbindlichkeit eingebüßt. Das schließt allerdings nicht aus, dass die in Jahrhunderten entwickelten Vorstellungen von Ehe und Familie heute psychologisch nachwirken und nach wie vor die gesellschaftliche Wirklichkeit beeinflussen. Vor diesem Hintergrund ergeben sich eine Reihe spannender Fragen, denen der Autor in seiner Untersuchung nachgeht. Eine davon ist die, ob und inwieweit im Bereich der Ehe gesetzte Rechtsnormen und das tatsächliche gelebte Leben einander entsprechen, auseinanderfallen oder sich wechselseitig beeinflussen. Dabei ist es das erklärte Ziel des Autors, einen historischen Prozess aufzuzeigen, der bis in die Gegenwart andauert, um so, angesichts einer langfristigen und fortdauernden Neuentwicklung von Ehevorstellungen, eine historische Tiefenperspektive zu gewinnen für den Blick auf die Gegenwart und auf diese Weise möglicherweise Orientierungshilfen zu finden für das Eherecht der Zukunft.

Der beeindruckende Umfang des Bandes von fast 1200 Seiten ist für eine Dissertation erstaunlich, entspricht aber durchaus der Größe des Themas, denn behandelt werden die persönliche Stellung von Mann und Frau im Recht der ehelichen Lebensgemeinschaft im deutschsprachigen Raum über einen Zeitraum von mehr als $200 \mathrm{Jah}-$ ren, von 1700-1914. Als Übergangszeit zwischen dem älteren Gemeinen Recht und dem neueren kodifizierten Recht gilt dem Autor das 18./19. Jahrhundert als der vielleicht aufschlussreichste Zeitraum der deutschen Rechtsgeschichte. Das ursprünglich gemeinrechtlich geprägte Eherecht im deutschsprachigen Raum entwickelte sich bis 1907 (Schweizer ZGB) immer mehr zum Gesetzesrecht. Im Zuge dieser Entwicklung kam es zeitweise zu einer weitgehenden Partikularisierung des Rechts in den deutschen Kleinstaaten. Mit dem Inkrafttreten des BGB 1900 gab es dann in Deutschland erstmals ein einheitliches gesetzliches Ehepersonenrecht. Die Abgrenzung 1914 wurde vom Autor gewählt, um die Auslegung des BGB-Eherechts durch die Gerichte bis zum Beginn des 1. Weltkrieges in seine Untersuchung mit einzubeziehen. Die Abgrenzung 1700 ermöglichte zum einen die Einbeziehung der Rechte und Entwürfe vor Inkrafttreten des PrALR, zum anderen die Einbeziehung der für das Eherecht zentralen Gedanken der Naturrechtler des 18. Jahrhunderts.

Nach der im ersten Teil erfolgten Klärung inhaltlicher und methodischer Fragen geht es im zweiten Teil des Buches um die "Grundlagen« der ehelichen Lebensgemeinschaft, $d$. h. vor allem um die im Bereich odes Eherechts-angewandten Rechte. Erfreulich ist, dass diese Grundlagen hier 
einmal nicht in eine Fußnote verwiesen oder in einem Halbsatz nur angedeutet werden. Stattdessen finden sich in diesem Kapitel ausführliche Darstellungen beispielsweise des "göttlichen Rechts", des Kanonischen Rechts, des römischen oder des Gemeinen Rechts. Dies gilt ebenso für die ausgewerteten Kodifikationen. Diese reichen vom Landrecht für das Königreich Preußen (PrLR) von 1721 über den bayrischen Codex Maximilianeus Bavaricus Civilis (CMBC) von 1756 bis zum Schweizer ZGB von 1907. Der Umfang des verarbeiteten Quellenmaterials ist beeindruckend, und so wird schon hier in den Grundlagen die Zersplitterung des deutschen Eherechts vor 1900 sozusagen zur Lektüreerfahrung. Der stringente Aufbau der Untersuchung, die klare, z. T. auch eigenwillige und persönliche Sprache des Autors, sein engagiertes Erkenntnisinteresse und die analytische Schärfe seiner Darstellung verhindern jedoch die Gefahr, in der Fülle des Materials "zu ertrinken «, und gewährleisten eine gute Lesbarkeit des durchweg spannenden Textes. Hierzu trägt auch der Anhang bei, in dem die wichtigsten Bestimmungen der z. T. wenig bekannten Gesetze sowie weitere zeitgenössische Stellungnahmen abgedruckt sind.

Im 3. Hauptteil des Bandes geht es dann um die persönlichen Rechte und Pflichten der Ehepartner im einzelnen, um die Pflicht zur Geschlechtsgemeinschaft (Leistung der ehelichen Pflicht), die Pflicht zur ehelichen Treue oder das Aufenthaltsbestimmungsrecht, die jeweils aufgeschlüsselt nach den unterschiedlichen Rechtsnormen behandelt werden. Untersucht wird, ob, wie weit und aus welchen Gründen die jeweilige Norm Männern und Frauen unterschiedliche Rechte und Pflichten zuwies und welche Konsequenzen dies hatte für das Eheleben. Dabei kommt der Autor zu dem Schluss, dass selbst dort, wo gleiche Rechte oder Pflichten auf der höchsten Definitionsebene normiert wurden, die abstrakte Gleichheit in der Praxis zu deutlichen Ungleichheiten führen konnte. Ein Beispiel ist ihm die gegenseitige Pflicht zur Geschlechtsgemeinschaft. Hier bestand die Ungleich heit darin, dass zum einen zumeist nur dem Mann ein Genuss an der ehelichen Geschlechtsgemeinschaft zugestanden wurde, in zeitgenössischen Gerichtsentscheidungen wurde dagegen ein eigenständiges Begehren der Frau häufig als unsittlich angesehen. Des weiteren wax nur für die Frau die Duldung der Geschlechtsgemeinschaft mit der Konsequenz von Schwangerschaften verbunden. Die dunkelste Seite dieser gegenseitigen Pflicht aber war für die Ehefrau die von fast allen Strafrechtlern der damaligen Zeit vertretene Rechtfertigung der Vergewaltigung in der Ehe als einer berechtigten Selbsthilfe des Mannes zur Durchsetzung seines sexuellen Forderungsrechts.

Im ersten und zentralen Abschnitt dieses dritten Teils geht es jedoch zunächst um die ausdrücklichen Vorrechte des Ehemannes gegenüber der Ehefrau, die der Autor unter dem Begriff »Eheherrschaft des Mannes« zusammenfasst. Zur Zeit der Geltung des Gemeinen Rechts wurde es allgemein als störend empfunden, dass die römischen Rechtsquellen die Ehe eher als außerrechtliche, soziale Tatsache auffassten, weshalb hier die Eheherrschaft ungeregelt blieb und keine Rechtsfiguren für die Regelung des persönlichen Verhältnisses der Eheleute entwickelt wurden. Für die Begründung der Herrschaftsrechte des Ehemannes wurde daher im deutschsprachigen Raum vor allem auf das kanonische Recht zurückgegriffen, wesentliche Rechtsquellen waren hier die Schöpfungsgeschichte des alten Testaments (1. Mose 3,16) sowie der Epheserbrief (Eph 5. 22-24), in welchem Paulus die Stellung des Mannes als Haupt der Frau vergleicht mit der Stellung Jesu als Haupt der Gemeinde. Galt den Kirchenrechtlern die Ehe als heiliges Sakrament, so wurde sie von den Naturrechtlern vor allem als ein Vertrag aufgefasst, bei dem die Eheherrschaft des Mannes Vertragsinhalt war. Umstritten war allerdings, ob eine Eheschließungserklärung, die nicht das Einverständnis der Frau in die Herrschaftsrechte des Mannes enthielt, eine Ehe überhaupt begründen konnte. Dies wurde insbesondere von Fichte bestritten, dessen Eherechtsphilosophie der Autor derjenigen Kants gegenüberstellt. Beide Philosophen, so die interessante Deutung Dunckers, behandelten unabhängig voneinander das Problem, inwieweit die Eheschließung mit einem Verlust der Menschenwürde verbunden sei. Für Fichte war dies nur ein Problem der Frau, da nur diese sich in der Ehe leidend zum Mittel der Befriedigung ihres Mannes mache. Tue sie dies aus Liebe zu ihrem Mann, so Fichtes Lösung, gewinne sie ihre Würde zurück. Kant dagegen sah das Problem für beide Parteien. Beide Ehepartneroerwarben nach seiner Eherechts- 
lehre mit der Eheschließung ein quasi dingliches körperliches Besitzrecht am anderen, der damit für den »Erworbenen « verbundene Würdeverlust konnte nach Kants Auffassung allerdings dadurch geheilt werden, dass der Erwerb des körperlichen Besitzrechts ein gegenseitiger war. Der Erwerb eines einseitigen Besitzrechts dagegen (wie bei Fichte) widersprach nach Kant der menschlichen Würde, nicht jedoch die Eheherrschaft des Mannes, sofern sie Ausdruck seines »natürlichen Vermögens « sei. Die trotz unterschiedlicher Formulierungen in allen Rechten, Kodifikationen und Entwürfen des untersuchten Zeitraums normierte potestas maritalis des Ehemannes wurde dann im BGB von 1896 zum »Entscheidungsrecht des Ehemannes in allen das gemeinschaftliche eheliche Leben betreffenden Angelegenheiten « ( $\$ 1354$ BGB, bis 1953 geltendes Recht). Die Motive begründeten dieses ehemännliche Vorrecht vor allem mit dem generalklauselähnlichen Begriff vom »Wesen der Ehe«. Dabei galt den Vätern des BGB das Entscheidungsrecht des Ehemannes als wesentliches, ehekonstituierendes Merkmal, das durch Vertrag nicht abdingbar war. Das BGB war dann auch das erste kodifizierte Recht, das von einer zahlenmäßig größeren Opposition, vor allem von der deutschen Frauenbewegung, wegen der Herrschaftsstellung des Mannes kritisiert wurde. Es gehört zu den Vorzügen der Untersuchung Dunckers, dass er sich mit den rechtskritischen Schriften der Feministinnen ausführlich auseinandersetzt - mit dem Ergebnis einer eher vorsichtigen Zustimmung. Zwar könne das BGB, gemessen an anderen Kodifikationen des Untersuchungszeitraums insoweit als gemäßigt gelten, als es das Entscheidungsrecht des Ehemannes auf die gemeinsamen ehelichen Angelegenheiten beschränke, es trug jedoch in das 20. Jahrhundert den Widerspruch hinein zwischen einer dem Recht der Minderjährigen angenäherten Rechtsstellung der Ehefrau und den im Allgemeinen Teil geschlechtsneutral formulierten Regelungen zur Rechtsund Geschäftfähigkeit.

Im 4. Kapitel untersucht der Autor dann die spannende Frage, inwieweit die geltenden rechtlichen Bestimmungen die Realität der geführten Ehen zwingend beeinflussen mussten oder ob Abweichungen von den gesetzlichen Regelungen durch Vertrag oder durch Verhalten möglich waren. Die wenigen nachgewiesenen Verträge, in de- nen die Parteien personenrechtliche, von der Eheherrschaft abweichende Regelungen trafen, etwa zum Wohnortbestimmungsrecht, konnten allerdings nach der ständigen Rechtsprechung des Reichsgerichts für den Mann keine Rechtsverbindlichkeit entfalten (so z. B. RGZ 61, 50, diese Entscheidung galt auch in den 50iger Jahren noch als Leitentscheidung zu $\$ 1354$ BGB). Eine Ehefrau hatte somit, wie der Autor überzeugend nachweist, keine Möglichkeit, sich vertraglich der Eheherrschaft zu entziehen. An dieser Stelle folgt dann eine der interessantesten Passagen des Buches, die Diskussion über die sogenannte freie Ehe zwischen der Frauenrechtlerin und ersten deutschen Juristin Anita Augspurg und der Schriftstellerin und Frauenrechtlerin Marianne Weber. Weber lehnte wie Augspurg die Eheherrschaft ab, plädierte aber, vielleicht vor dem Hintergrund ihrer eigenen Ehe mit Max Weber, dafür, Reformen anzustreben und bis dahin zu versuchen, innerhalb der Ehe anders zu leben, als die Gesetze es nahe legten. Augspurg dagegen erklärte 1905 in einem offenen Brief, einer »Frau von Selbstachtung « bliebe nach ihrer Auffassung nur die Möglichkeit einer freien Ehe offen, da selbst bei dem erklärten Willen der Ehepartner, durch abweichendes Verhalten eine herrschaftsfreie Ehe zu führen, folgende durch individuelles Verhalten nicht beeinflussbare Faktoren bestehen blieben: 1.) das Verhalten Dritter (bei Schulanmeldung der Kinder oder Rechtsgeschäften jeder Art); 2.) die direkten gesetzlichen Folgen (Aufgabe des eigenen Namens, Verlust wesentlicher Elternrechte); 3.) die Rechtlosigkeit gegenüber dem Ehemann, die bewirke, dass eine gleichberechtigte Beziehung allein von seinem guten Willen abhängig sei. Der Autor folgt hier weitgehend Augspurgs Argumentation, glaubt aber, dass diese die Folgen zu wenig bedenke, welche die Aufgabe der Schutzbestimmungen des Eherechts für den wirtschaftlich und persönlich schwächeren Teil habe. Dieses Argument scheint mir allerdings dadurch entkräftet zu sein, dass Augspurg ausdrücklich nur diejenigen Frauen aufforderte, eine freie Ehe zu führen, die es sich persönlich und wirtschaftlich leisten zu können glaubten, durch ihr Vorbild auf eine Gesetzesänderung hinzuwirken.

Im 5. Teil des Buches folgt eine kurze Darstellung der W Wechselbeziehungen zu verwandten Rechtsgebieten (eheliches Güter- 
recht, Kindschaftsrecht u. a.), im abschließenden 6. Teil geht es dann nicht zuletzt um einen Ausblick auf das Eherecht der Gegenwart und der Zukunft. Duncker vertritt hier die Auffassung, dass keine der heute gültigen Ehedefinitionen der Realität moderner, zeitgenössischer Ehen gerecht werde, da diese vor allem gekennzeichnet seien durch die fortschreitende Auflösung ehebestimmender Merkmale. Er stellt deshalb drei, von ihm aus dem historischen Diskurs entwickelte, neue Ehedefinitionen zur Diskussion (251, 1114, Fn. 4813): » Ehe ist das, was das am jeweiligen Ort geltende Recht als Ehe anerkennt (machtpositivistische Definition anhand von Gedanken Hobbes); b.) Ehe ist die innige Gemeinschaft zweier Menschen, die keinen Zweck außerhalb ihrer selbst hat und ihr eigener Zweck ist (weiterentwickelt auf der Basis von Fichte); c.) Ehe ist die Verbindung zweier Menschen zu ungeteilter Lebensgemeinschaft (weiterentwickelt auf der Basis des römischen Rechts).«Ob diese Definitionen hilfreich sind für das Eherecht der Zukunft, mag dahin gestellt bleiben, sicher ist jedoch, dass diese Untersuchung neue Maßstäbe setzt für die vergleichende rechtsgeschichtliche Forschung. Dass das deutsche Eherecht bis fast in die Gegenwart hinein patriarchalisch war, ist ja nichts Neues, doch zeigt erst der hier unternommene Vergleich des frühen deutschsprachigen Gesetzesrechts mit biblischen, römischen und kirchenrechtlichen Quellen sowie mit dem Gemeinen Recht, wie sehr die Eherechtsgesetzgebung des 18. und 19. Jahrhunderts Teil eines überregionalen, langfristigen, rechtlichen und außerrechtlichen Diskurses war, innerhalb dessen der gesetzgeberische Gestaltungsspielraum kaum genutzt wurde. Und so wird am Ende des Buches sehr deutlich, wie klein der historische Zeitraum von gut 50 Jahren ist, seit mit der Außerkraftsetzung des $\ 1354$ BGB der Gleichheitsgedanke allmählich auch für die rechtliche Gestaltung des persönlichen Eherechts bestimmend wurde.

\section{Christiane Henke}

Ralf Poscher, Grundrechte als Abwebrrechte - Reflexive Regelung rechtlich geordneter Freibeit, Tübingen (Mobr Siebeck, Jus Publicum 98) 2003, 445 S., 94,- $€$.

In der Rechtsprechung des BVerfG wird den Grundrechten neben ihrer kassischen
Funktion als Abwehrrechte gegen staatliche Eingriffe auch ein objektiv-rechtlicher Wertgehalt zugesprochen. Diesen hat das Gericht verschiedentlich herangezogen, um weitere Grundrechtsfunktionen zu entwickeln: $\mathrm{Zu}$ nennen sind neben den Schutzpflichten vor allem die Leistungsund Teilhaberechte und die institutionellen Gewährleistungen. Besondere Bedeutung hat in der Rechtsprechung auch die Ausstrahlungswirkung der Grundrechte in das Privatrecht erlangt.

Ungeachtet der funktionalen Fortentwicklung hat das BVerfG aber stets die abwehrrechtliche Dimension der Grundrechte hervorgehoben. So formuliert das Gericht bereits in seiner Lüth-Entscheidung aus dem Jahr 1951: »Ohne Zweifel sind die Grundrechte in erster Linie dazu bestimmt, die Freiheitssphäre des einzelnen vor Eingriffen der öffentlichen Gewalt zu sichern; sie sind Abwehrrechte des Bürgers gegen den Staat $\ll .1$

An dieses Bekenntnis des BVerfG zur abwehrrechtlichen Funktion der Grundrechte knüpft Ralf Poscher in seiner Berliner Habilitationsschrift an. Die Arbeit will ein modernes Verständnis des Abwehrrechts entwickeln, das dieses methodisch anschlussfähig macht und der verschiedentlich geübten Kritik an der abwehrrechtlichen Betrachtungsweise Rechnung trägt. Poscher will nachweisen, dass über ein abwehrrechtliches Verständnis auch solche Konstellationen zufriedenstellend gelöst werden können, für die zum Teil andere funktionale Ansätze entwickelt wurden (S. 2). Deshalb unternimmt er den Versuch, die gesamte Grundrechtsdogmatik auf ein abwehrrechtliches Verständnis zurückzuführen. Auf diesem Wege will er die anderen in der Literatur und Rechtsprechung entwickelten Grundrechtsfunktionen überflüssig machen. Neben dem Abwehrrecht möchte Poscher andere Funktionen nur anerkennen, wenn sie der Verfassungstext (wie z. B. in Art. 1 Abs. 1 S. 2, Art. 6 Abs. 4 und Art. 6 Abs. 5 GG) ausdrücklich vorsieht (S.96f.). Durch die Rückbesinnung auf das Abwehrrecht soll die aus der Sicht des Autors zunehmende »Konstitutionalisierung der Rechtsordnung « (S. 14) und die Gefahr eines »Verfassungsjurisdiktionsstaates « (S.410), dogmatisch aufgefangen und eingedämmt werden. 
Zur Begründung des modernen Verständnisses der Abwehrfunktion dient Poscher vor allem der von ihm selbst entwickelte Topos von der Reflexivität der Grundrechte. Hierzu stellt er in der Einleitung fest: »Die Arbeit legt einen Schwerpunkt darauf, die Grundrechte als reflexive Rechte $\mathrm{zu}$ verstehen. Mit der Reflexivität der Grundrechte ist zunächst nichts weiter als die Trivialität gemeint, dass die Abwehrrechte sich in erster Linie an den regelnden Staat richten. Die Abwehrrechte regeln Konflikte, die in der Gesellschaft auftreten, nicht unmittelbar selbst. Unmittelbar binden die Grundrechte den Staat dagegen nur bei der Regelung von Konflikten, die grundrechtlich geschützte Freiheits- und Gleichheitsinteressen der Grundrechtsträger berühren« (S. 2).

$\mathrm{Zu}$ Beginn der Untersuchung legt der Autor ausführlich sein methodisches Vorverständnis dar (S. 5 ff.). Gegenüber dem Dezisionismus erkennt Poscher den Wert eines dogmatischen Systems vor allem in seiner stärkeren Systematisierung und Berechenbarkeit, die bei einer einzelfallbezogenen Methode naturgemäß nicht gegeben ist. Es genügt seiner Ansicht nach aber nicht, ein »stimmiges System begrifflicher Definitionen und Verknüpfungen « zu entwerfen. Vielmehr müsse das dogmatische System in der Lage sein, sowohl »unser gemeinsames Vorverständnis bezüglich einzelner Grundrechtsnormen «, als auch die wesentlichen Ergebnisse der Rechtsprechungspraxis abzubilden. Denn je häufiger und je weiter ein dogmatisches System von der etablierten Praxis abweiche, desto höher werde die Begründungslast (S. 13). Diese methodischen Ausführungen dienen Poscher als Anleitung für seine weitere Untersuchung: Das zu entwickelnde Grundrechtsverständnis soll alle wesentlichen, in der Rechtsprechung entwickelten Konstellationen erfassen und sich somit nicht nur in der Wissenschaft, sondern auch gegenüber der forensischen Praxis behaupten. Damit gewinnt insbesondere die Rechtsprechung des BVerfG eine wesentliche Bedeutung.

Zunächst jedoch beschäftigt sich der Autor in einem sehr lesenswerten Abschnitt (S. 15 ff.) mit den ideengeschichtlichen Grundlagen des Abwehrrechts und seiner historischen Entwicklung in Deutschland über die Paulskirchenverfassung, den Spätkonstitutionalismus, die Weimarer Republik bis hin zur frühen Verfassungsent- wicklung in der Bundesrepublik. Zusammenfassend stellt Poscher fest, dass sich die abwehrrechtliche Funktion der Grundrechte erst unter der Rechtsprechung des BVerfG in der »klassischen « Ausprägung entwickelt hat, wie wir sie heute u. a. mit der Schrankendogmatik und dem Grundsatz der Verhältnismäßigkeit verbinden (S. 47).

Im folgenden Abschnitt "Stand der grundrechtsdogmatischen Diskussion«(S. 72 ff.) setzt sich Poscher u. a. ausführlich mit der Prinzipientheorie der Grundrechte auseinander, die maßgeblich von Robert Alexy und dessen Schülern entwickelt wurde. Poscher kritisiert die dogmatische "Unbestimmtheit « und »Strukturlosigkeit « des prinzipientheoretischen Ansatzes in sehr deutlicher, teilweise auch polemischer Form (»Dem prinzipientheoretischen Ansatz ist nichts fremd «, S. 76, »Nullpunkt der Dogmatik «, S. 81). Er weist insbesondere darauf hin, dass der kategoriale Unterschied zwischen Regeln und Prinzipien in der von den Prinzipientheoretikern apostrophierten Weise nicht haltbar sei (S. 78 ff.). Aber auch einem Funktionenpluralismus, bei dem die verschiedenen Grundrechtsfunktionen nebeneinander stehen, erteilt Poscher eine Absage. Denn bevor man für jedes neue Grundrechtsproblem eine neue Grundrechtsfunktion entwickle, müsse geprüft werden, ob die jeweilige Fallkonstellation nicht bereits durch die klassische Abwehrfunktion befriedigend gelöst werden kann (S. 88).

Nach einer Auseinandersetzung mit den freiheits- und staatstheoretischen Grundlagen des Abwehrrechts (S. $106 \mathrm{ff}$.) entwickelt Poscher im folgenden Kapitel seine Feststellungen zur »Totalität und Reflexivität« des Abwehrrechts (S. 153 ff.). In den Mittelpunkt der Betrachtung stellt er dabei mehrpolige Rechtsverhältnisse, in denen »der Staat durch den regelnden Eingriff in die Rechte des einen Grundrechtsträgers dessen Verhältnis zu einem anderen Grundrechtsträger gestaltet «(S. 153). Diese Konstellation ist klassischerweise im Privatrecht anzutreffen. Poscher zeigt, dass sich die sog. mittelbare Drittwirkung (oder Ausstrahlungswirkung) der Grundrechte im Privatrechtsverhältnis abwehrrechtlich erfassen lässt, wenn man die Reflexivität der Grundrechte in Rechnung stellt. Der Staat ist aufgrund seines rechtsstaatlichen Gewaltmonopols dazu verpflichtet, die Interessenkonflikte $2 \mathrm{der}$ :Grundrechtsträger 
untereinander umfassend $\mathrm{zu}$ regeln (S.148f.). Eine solche Regelung ist aber zwangsläufig mit einem Grundrechtseingriff verbunden, da zumindest die Freiheit eines Rechtsunterworfenen beschränkt wird. Dies illustriert Poscher an einem konkreten Fall aus der Rechtsprechung des BVerfG, der einen mietrechtlichen Streit um die Anbringung einer Satellitenempfangsanlage betrifft: Wird der Eigentümer durch das Mietrecht gezwungen, die Installation der Empfangsanlage durch den Wohnungsmieter zu dulden, so liegt darin ein Grundrechtseingriff zu Lasten des Eigentümers. Wäre eine solche Duldungspflicht aber verfassungswidrig (etwa weil sie den Eigentümer unzumutbar schwer belastet), so kann der Konflikt von der Rechtsordnung nicht offengelassen werden. Dann müsste eine Regelung in Richtung auf eine Unterlassungspflicht des Mieters erfolgen. Der Grundrechtseingriff läge dann auf der anderen Seite (S. 188). Wie sich das Recht auch entscheidet, sei es zugunsten des Eigentümers, sei es zugunsten des Mieters, in jedem Fall sind die Grundrechte betroffen, in jedem Fall liegt ein Grundrechtseingriff vor. Tertium non datur. Darum geht es Poscher, wenn er von »Totalität« und »Reflexivität« des Abwehrrechts in mehrpoligen Rechtsverhältnissen spricht. Mit Recht hebt Poscher dabei die Staatsgerichtetheit der Freiheitsrechte hervor. Die Abwehrrechte regeln nicht den Konflikt der Grundrechtsträger; sie haben keine unmittelbare Wirkung unter Privaten. »Sie regeln vielmehr nur die Regelung des Konflikts durch die gesetzgebende, rechtsprechende und vollziehende Gewalt« (S. 226).

In einem folgenden Kapitel (S. 227 ff.) weist Poscher im Wege einer umfangreichen Analyse der Rechtsprechung nach, dass das BVerfG auch im Rahmen seiner Entscheidungen zur mittelbaren Drittwirkung an der Staatsgerichtetheit der Grundrechte festhält. Das Gericht hat die Grundrechte als staatsgerichtete Abwehrrechte über die Figur der mittelbaren Drittwirkung aktualisiert, um die Distanz zu der Lehre von der unmittelbaren Grundrechtsbindung der Privatrechtssubjekte deutlich zu machen. Bei der Anwendung der Gesetze sind die Zivilgerichte an die Grundrechte gebunden. "Diese unmittelbare Bindung der rechtsanwendenden Gewalten bei der Konkretisierung gesetzlicher Vorschriften meint das BVerfG, wenn es bezüglich der
Zivilrichter und der übrigen Fachgerichte von dem Erfordernis der Auslegung im Lichte der Grundrechte, der Beachtung der Ausstrahlungswirkung, der Wechselwirkung und der mittelbaren Grundrechtswirkung spricht« (S. 280). Insofern stellt sich die Ausstrahlungswirkung der Grundrechte als nichts anderes dar als eine verfassungsorientierte Auslegung des einfachen Rechts (S.281). Diese Feststellung beschränkt sich nach Poscher nicht nur auf das Privatrecht, sondern gilt auch für andere Dreieckskonstellationen, etwa im Rahmen von drittbelastenden Genehmigungsverfahren, im Strafrecht oder bei der Regelung von Konkurrenzverhältnissen (S. 289 ff.).

In einem letzten Teil entwickelt Poscher aus den gewonnenen Erkenntnissen eine »Dogmatik des reflexiven Abwehrrechtsverständnisses « (S. 315 ff.). Die Vorteile dieses Ansatzes liegen seiner Ansicht nach darin, dass die Staatsgerichtetheit der Grundrechte wieder verstärkt in das Blickfeld der Grundrechtsprüfung geführt werden. Bezugspunkt für die abwehrrechtliche Prüfung sei eben nicht der zu regelnde Konflikt von Privatrechtssubjekten, sondern die staatliche Regelung und deren Regelungszweck. Der Konflikt selbst wird nicht grundrechtlich entschieden, sondern nur die staatliche Konfliktregelung (durch Gesetzgebung, Verwaltung oder Rechtsprechung) auf ihre Grundrechtskonformität hin überprüft. Abschließend stellt Poscher fest: »Indem sich die abwehrrechtliche Dogmatik von der Vorstellung befreit, Konflikte zwischen Grundrechtsträgern fänden ihre Lösung in der Abwägung unterschiedlicher Grundrechtspositionen, befreit sie sowohl die politische als auch einfachrechtliche Argumentation von einem umfassenden verfassungsrechtlichen Zugriff«(S. 408).

Der unbestreitbare Verdienst von Poschers Arbeit liegt darin, den Nachweis geführt zu haben, dass die sog. mittelbare Drittwirkung der Grundrechte in der Rechtsprechung weniger als eine neue, objektive Grundrechtsfunktion entwickelt wurde, sondern vielmehr als eine besondere Ausprägung ihrer klassischen Abwehrfunktion. Wer sich mit der Rechtsprechung des BVerfG intensiver befasst, den kann diese Feststellung keineswegs überraschen. Schließlich hat das Gericht schon in der Lüth-Entscheidung die Grundrechtsbin- 
dung des Zivilrichters hervorgehoben, ${ }^{2}$ freilich unter gleichzeitiger Betonung derin der Tat missverständlichen-»objektiven Werteordnung «.

Gegen die dogmatischen Schlussfolgerungen, die Poscher aus seinem Verständnis der Grundrechte als reflexive Abwehrrechte zieht, lassen sich vielfältige Fragen und Einwendungen vorbringen. Selbstverständlich kann im Rahmen dieser Besprechung keine grundlegende Kritik geführt werden. Dennoch sollen im Folgenden zwei Punkte näher beleuchtet werden, die Kritik herausfordern.

Zum Einen: Entsprechend seinem abwehrrechtlichen Grundverständnis möchte Poscher den Vorbehalt des Gesetzes auch auf das Privatrecht anwenden. D.h. die Zivilgerichte dürfen einen privatrechtlichen Konflikt nur dann entscheiden, wenn sie sich für ihre Entscheidung auf eine hinreichende gesetzliche Grundlage stützen können. Diesem Gesetzesvorbehalt sollen nach Poscher auch die zivilrechtlichen Generalklauseln genügen.

Es erscheint bereits fraglich, welchen Zweck der Gesetzesvorbehalt im Privatrecht überhaupt erfüllen kann. Die Generalklauseln jedenfalls können ob ihrer Weite und Unbestimmtheit (»Sittenwidrigkeit«, »Treu und Glauben«, »Verletzung eines sonstigen Rechts «) kaum die notwendige Begrenzungsfunktion entfalten. Dem lässt sich auch nicht mit dem Einwand begegnen, das Bürgerliche Recht würde nur wenige Generalklauseln enthalten und ansonsten eine hohe Regelungsdichte aufweisen (S. 334). Denn eine solche rein formalquantitative Sichtweise lässt außer Betracht, dass der große Teil der vertraglichen Inhaltskontrolle über die Generalklauseln der $\int \mathbb{S} 138,242,307$ BGB erfolgt und auch das Deliktsrecht durch die wenig bestimmte Zentralnorm des $\ 823$ BGB beherrscht wird. In der Praxis kommt gerade diesen Vorschriften überragende Bedeutung zu, und gerade hier ist ja auch die Einwirkung der Grundrechte virulent. Poscher geht falsch in der Annahme, das Problem der eingriffsrelevanten Generalklauseln sei ein »allgemeines, das einem im Polizei- und Ordnungsrecht, Datenschutzrecht und anderen Gebieten des besonderen Verwaltungsrechts ebenso begegnet wie im Privatrecht «(S. 334). In den zuletzt genannten Fällen haben die Generalklauseln heute ne- ben den speziellen Vorschriften lediglich eine Auffangfunktion. Das ist jedenfalls für die ordnungsrechtliche Generalklausel kaum bestreitbar. Dort wird übrigens der Begriff der »öffentlichen Ordnung « mit Recht eng ausgelegt. »Treu und Glauben « werden aber von der Rechtsprechung extensiv angewendet. Die Generalklauseln des Privatrechts und des öffentlichen Rechts sind daher weder in ihrer Funktion noch in ihrem Regelungsgehalt miteinander vergleichbar. Die Spielräume im Privatrecht sind sehr viel größer.

Nach den Ausführungen Poschers bleibt im Übrigen unklar, wie in dem Fall verfahren werden soll, dass für die Entscheidung eines privatrechtlichen Rechtsstreits keine einfachrechtlichen Vorschriften vorhanden sind. Auf die Problematik im Arbeitskampfrecht hat der Autor selbst hingewiesen. Wie Poscher an anderer Stelle zu Recht feststellt, sind die Gerichte aufgrund des Gewaltmonopols und des Justizgewährleistungsanspruchs verpflichtet, den ihnen vorgelegten Rechtsstreit $\mathrm{zu}$ entscheiden. Wie aber ist zu entscheiden, wenn eine gesetzliche Regelung im positiven Recht nicht aufzufinden ist, auf die sich das Gericht stützen könnte? Im öffentlichen Recht wäre der Fall klar: Eine staatliche Maßnahme, die ohne ausreichende Gesetzesgrundlage in Freiheitsrechte des Bürgers eingreift, ist unzulässig. Im Streit zwischen Privaten gibt es eine solche Entscheidungsregel aber nicht. Es kann sie nicht geben.

Die propagierte Bindung der Zivilrechtsprechung an den Gesetzesvorbehalt hat aber noch eine andere, weitaus problematischere Konsequenz: Macht man mit der Forderung von Poscher ernst, so wäre damit jede Rechtsfortbildung praeter legem von vornherein ausgeschlossen. Dann hätten die Zivilgerichte beispielsweise nicht bei schwerwiegenden Verletzungen des Persönlichkeitsrecht gegen den ausdrücklichen Wortlaut des \$253 BGB eine Geldentschädigung auch für immaterielle Schäden zusprechen dürfen. ${ }^{3}$ Die von Poscher aufgestellte Forderung, den Gesetzesvorbehalt auf das Privatrecht auszuweiten, steht damit im Widerspruch zur Soraya-Entscheidung des BVerfG. Dort wird die Befugnis der Zivilgerichte zur »schöp- 
ferischen Rechtsfindung « ${ }^{4}$ ausdrücklich anerkannt. Entgegen seiner eigentlichen Intention, den Zivilgerichten einen größeren Freiraum gegenüber dem Verfassungsrecht einzuräumen, schlägt Poscher ihnen über den propagierten Gesetzesvorbehalt die Möglichkeit einer übergreifenden Rechtsfortbildung aus der Hand.

Widerspruch fordern auch die Ausführungen über die Gleichheitsrechte heraus. Nach Auffassung Poschers sind sie der "neuralgische Punkt der klassischen Diskussion um das Verhältnis von Grundrechten und Privatrecht «(S. 337). Dabei beziehe die Frage ihre Spannung weniger aus der Kontroverse um die Antwort. Denn im Ergebnis herrsche weitgehend Einigkeit: Eine Bindung der Privatrechtssubjekte an den allgemeinen und die speziellen Gleichheitssätze komme nicht in Betracht (S. 337). So behauptet Poscher: »Als staatsgerichtete Rechte schützen die Gleichheitsrechte nicht davor, durch andere Grundrechtsträger ungleich behandelt zu werden. Entsprechend verbieten die Gleichheitsrechte keine Regelungen, die den Grundrechtsträgern die Möglichkeit gegenseitiger Ungleichbehandlung einräumen« (S.339). Wenn der Zivilrichter in seinem Urteil beispielsweise die Regelung im Testament sanktioniere, wonach die Tochter des Erblassers aufgrund ihres Geschlechts vom Erbe ausgeschlossen wird, so liege darin kein Eingriff in Art. 3 Abs. 3 GG. Das Gericht knüpfe seine Entscheidung selbst nicht an eine bestimmte Geschlechtszugehörigkeit an, sondern lasse nur eine Diskriminierung unter Privaten zu (S. 340). Die speziellen Gleichheitssätze entfalten nach Poscher keine mittelbare Drittwirkung in das Zivilrecht: "So kann aus Art. 3 Abs. 3 GG für \138 Abs. 1 BGB nicht gefolgert werden, dass eine Diskriminierung, die Art. 3 Abs. 3 GG im Staat-Bürger-Verhältnis verbietet, im Privatrechtskontakt zweier Bürger gegen die guten Sitten verstößt « (S. 342).

Schon die einleitende Feststellung Poschers, über die Bedeutungslosigkeit der Gleichheitsgrundrechte im Privatrecht herrsche »weitgehend Einigkeit «, verwundert. Soweit erkennbar, wird die Ausstrahlungswirkung zumindest des speziellen Gleichheitssatzes nach Art. 3 Abs. 3 GG in der Kommentarliteratur (nahezu) einhellig befürwortet. ${ }^{5}$ Auch das BVerfG hat in seiner Rechtsprechung immer wieder betont, dass die Grundrechte über die Generalklauseln auf das Privatrecht einwirken, und hat insofern bezüglich der Gleichheitsrechte keine Ausnahme gemacht. Wie Poscher an anderer Stelle selbst ausführt, sind die Gleichheitsrechte ihrem Wesen nach ebenfalls Abwehrrechte gegen staatliches Handeln: »Die Ungleichbehandlung muss nur als Eingriff in das Recht auf Gleichbehandlung verstanden werden. (...) Wenn ein Verhalten in das Recht auf Gleichbehandlung eingreift und kein zureichender Grund vorliegt, dann soll das Verhalten unterlassen werden« (S. 157). So gesehen besteht kein Grund, die Gleichheitsrechte in ihrer Ausstrahlungswirkung anders zu behandeln als die Freiheitsrechte. Wenn das Zivilgericht durch sein Urteil eine testamentarische Regelung billigt, durch die der Erblasser seine Tochter aufgrund ihres Geschlechts benachteiligt, dann liegt in der Entscheidung des Gerichts selbst zwar keine Ungleichbehandlung. Das Urteil erlegt der Tochter jedoch die Pflicht auf, die Diskriminierung ihres verstorbenen Vaters zu erdulden. Insoweit wird der Freiheitsraum des Art. 3 Abs. 3 GG durch die staatliche Duldungspflicht verkürzt. Der Fall liegt nicht anders, als wenn das Zivilgericht aus Gründen der Meinungsfreiheit einem Betroffenen die Hinnahme eines Presseberichts zumutet, in dem ehrbeeinträchtigende Behauptungen enthalten sind. Auch hier tätigt das Gericht die Äußerungen ja nicht selbst, sondern es sanktioniert durch die Entscheidung die Ehrbeeinträchtigung und weist den Betroffenen an, diese zu dulden.

Übrigens hat der $\mathrm{BGH}$ die mittelbare Drittwirkung des Art. 3 Abs. 3 GG gerade für den von Poscher angeführten Erbrechtsfall ausdrücklich anerkannt. ${ }^{6}$ Das Verdikt der Sittenwidrigkeit will der BGH jedoch mit Rücksicht auf die konkurrierende Testierfreiheit (Art.14 Abs. 1 S. 1 GG) auf begrenzte Ausnahmefälle beschränken. Das ist die dogmatisch richtige Lösung. Die von Poscher vorgenommene Differenzierung zwischen Freiheits- und Gleichheitsrechten, die sich allein am Ter-

5 Statt vieler: Osterloh, in: Sachs, GG 3. Aufl. 2002, Art. 3 Rn. 233; Jarass, in: Jarass/Pieroth, GG 6. Aufl. 2002, Art. 3 Rn. 119.

6 V́gl. BGH NJW 1999, 566(569) - Hohenzollerntestament. 
minus der »Ungleichbehandlung « festmacht, ist hingegen rein konstruktivistisch und daher abzulehnen.

\section{Michael Wrase}

Günter Frankenberg/Peter Niesen (Hrsg.), Bildverbot. Recht, Ethik und Ästhetik der öffentlichen Darstellung, Münster (LIT Verlag) 2004, 194 S., 17,90€.

\section{I.}

Der durchschnittlich informierte, aufmerksame und verständige Volljurist hat vermutlich schon mehrmals in seinem Leben ein Kunstmuseum besucht. Egal in welchem Kunstmuseum er war, er wird dort spätestens im Museumsshop auf René Magrittes Bild "La trabison des images" (1929) bzw. ein Plakat dieses Bildes gestoßen sein. Ich brauche vermutlich nicht mit Worten zu wiederholen, was das Bild zeigt. Sie wissen schon, es geht um die Pfeife, die keine sein soll. Nicht nur, weil das Bild inzwischen zu einer klassisch gewordenen Anthologie der modernen Bilder gehört, auch weil der Jurist mit der Dynamik von Bedeutung bestens vertraut ist, wird ihn dieses Bild vermutlich nicht mehr irritieren als andere Dinge. Das Bild liefert ihm Zeichenkritik - es erinnert ihn daran, dass Schein und Sein von Zeichen immer mal wieder auseinander und Begriff und Bild immer mal wieder ineinander fallen. ${ }^{1}$ Wie steht es da mit der Macht der Bilder? Mit einer Vorstellung von magischen Bildmächten verträgt sich Magrittes Sprachbild schon deswegen nicht, weil es sich eben um ein Sprach-Bild handelt. Ob Verrat oder Macht: alles ist wie die Schönheit im lesenden Betrachter. Um vom Bild nicht beherrscht zu werden, braucht der Betrachter nur zum Bildalphabeten zu werden. Bilder zwingen zu nichts, außer zur Bildung. Wie alles, so ist auch das Bild etwas, das als etwas gelesen werden soll. Diese Lese-, Interpretations-, und Kommentierungszwänge als Beherrschungsstrategie sichtbar zu machen, war wohl die Passion der Moderne, und sie pflegte diese Leidenschaft mit der Markierung immer weiterer ästhetischer Differenzen zwischen Text und Kommentar oder Zeichen und Referenz. Jede Markierung einer Differenz ent- täuschte über angenommene Bildmächte. Das »offene Kunstwerk « oder die »offene Gesellschaft der Verfassungsinterpreten « waren die wohl jüngsten Versuche, der Operation der Differenzierung einen Eigennamen zu geben und so auf politische Einheitsbildung unter den Bedingungen der Pluralität zu beziehen. ${ }^{2}$

Ist es dann ein Widerspruch, dass man bisher von noch keinem Gerichtsurteil gehört hat, in dem die Ansprüche einer monegassischen Prinzessin mit den Worten abgewiesen wurden: »Ceci n'est pas Caroline«? Die Antwort hängt wohl davon ab, mit welchen Elementen und Einheiten wir unsere Vorstellung von Gesellschaft und Öffentlichkeit chiffrieren. Der durchschnittlich informierte, aufmerksame und verständige Volljurist überträgt seine Annahmen aus dem Museum in der Regel nicht in den Gerichtssaal. Er kann vor Magritte ein aufgeklärter Bildkritiker sein und vor Caroline an seinem Glauben an die Macht der Bilder festhalten. Ignoriert er damit die Zeichen-, Bild- und Medientheorien des 20. Jahrhunderts oder ist er in seiner Kunst, Bilder von Bildern zu unterscheiden, gerade konsequent modern? Vielleicht ist gerade die Möglichkeit zur Ignoranz modern? Schließlich lieferte die Moderne mit ihrem Verständnis von der Lesbarkeit der Welt auch die Vorstellung, dass Bilder zeigen, indem sie verbergen. ${ }^{3}$ So kann der Einzelne sich eben in verschiedenen offenen Gesellschaften bewegen - seine Ideen moderner Bilder müssen sich nicht mit seinen Ideen »öffentlicher Darstellungen« berühren.

Will man an der Idee einer Einheit des Wissens festhalten, so ist das nicht besonders befriedigend. In der Rechtswissenschaft verbreitet sich daher nun das Unbehagen, auf die Bedingungen der neuen Medien und die Konjunktur der Massenmedien nicht adäquat reagieren zu können. In diesem Sinne gibt es eine doppelte Ikonophobie der Jurisprudenz. Auf einer Ebene ist das methodische Ideal der nachhumanistischen

2 Umberto Eco, Das Offene Kunstwerk, Frankfurt a.M. 1968; Peter Häberle, Die offene Gesellschaft der Verfassungsinterpreten, JZ 1975, S. S. 297 ff.; zum Ende der Offenheit u. a.: Friedrich Kittler/ Jaques Derrida, Politik des Eigennamens, Berlin 2000; zu den Paradoxien von Geschlossenheit und Offenheit: Armin Nassebi, Geschlossenheit und Offenheit. Studien zur Theorie der modernen Gesellschaft, Frankfurt a.M. 2003.

37 Marin Fieidegger, Die Technik und die Kehre, Pfullingen 1962, S. 25.
1 Differenzierter hierzu: Michael Foucault, Dies ist keine Pfeife, München 1997. 
Rechtswissenschaft bilderfeindlich geworden. ${ }^{4}$ Auf einer anderen Ebene schließen sich heute diverse Autoren in der Rechtswissenschaft der Diagnose der »Bilderflut « oder des "Iconic Turn « an. ${ }^{5}$ Es wird die Vorstellung formuliert, die "auf der Basis von gedruckter Schrift entwickelten Konzeptionen der Kommunikationsfreibeit" erschienen "angesichts der Medienentwicklung im post-gutenbergschen Zeitalter überbolt«. ${ }^{6}$ Hierzu findet sich die Diagnose, die entscheidenden Juristen würden die Bedingungen visueller Kommunikation nicht hinreichend reflektieren, um Bilder noch adäquat beurteilen zu können. ${ }^{7}$

\section{II.}

Dies ist der Ausgangspunkt des von Günter Frankenberg und Peter Niesen herausgegebenen Sammelbandes zu Bilderverboten. Hervorgegangen aus einer Vorlesungsreihe zur Kommunikationsfreiheit der Johann-Wolfgang Goethe-Universität Frankfurt versammelt der Band Texte aus verschiedenen Fachdisziplinen zu der Frage ob, wie oder welche Bilder verboten werden sollen. Der öffentliche Bilderstreit der letzten 20 Jahre wird entfaltet: Von den Aufnahmen aus dem Beau Rivage in Genf (Barschel) über die Benettonkampagne hin zu den Plastinationen der »Körperwelten « und Bildern der einstürzenden Doppeltürme reicht die Spannbreite der Darstellungen, die Zensurimpulse auslösten.

Günter Frankenberg leitet den Band mit einem Text über den normativen Blick ein. Frankenberg erinnert an einen unhintergehbaren Punkt des Bilderstreites: So wie der Betrachter zu der Deutung eines Bildes gezwungen ist, so ist die Gesellschaft zur Bewertung von Bildern gezwungen: Der

4 Fabian Steinhauer, Who's afraid of black, red and gold. Zur Geburt der Ikonophobie aus dem Geist des Kriegsrechts, in: Werner Ernst (Hrsg.), Aufspaltung und Zerstörung durch disziplinäre Wissenschaften, Salzburg 2002.

5 Es gibt bereits eine Bücherflut zum Topos der Bilderflut und zahlreiche Schriften zum Topos des Iconic Turn. Grundlegend: William T.J. Mitchell, Picture Theory, Chicago 1994; Gottfried Boehm (Hrsg.), Was ist ein Bild?, München 1994; Harald Szeemann (Hrsg.) Katalog zur documenta 5, Kassel 1972.

6 So die Herausgeber in der Einleitung.

7 So vor allem Klaus Röhi, Das Recht nach der visuellen Zeitenwende, IZ 2003, S. 339. normative Blick verbrüdert sich mit dem deutenden Blick. Gegen eine vorschnelle Verbrüderung erinnert Frankenberg an die analytische Trennung von Ethik, Ästhetik und Recht des Bilderverbotes (7). Er will damit den kollidierenden Gesetzmäßigkeiten des Bilddiskurses zwischen bildnerischer und personaler Autonomie gerecht werden. Die analytische Trennung soll juridisch klären, was durch Begriffe mit »extralegaler Herkunft« verunreinigte Maßstäbe brachte (16). Frankenberg weist dabei zugleich auf das Kernproblem dieser analytischen Trennung: Die Korrespondenz ethischer, ästhetischer und rechtlicher Maßstäbe ist nur schwer zu entschlüsseln. Gleichwohl - so Frankenberg - halte die Eigengesetzlichkeit des Ästhetischen Abstand zu den Gesetzen des Rechtes, und das Recht respektiere in liberalen Gesellschaften die Geltungssphäre der Kunst; es sei ein Gebot der Klugheit, dass sich über Geschmack nicht streiten lasse. In der Geschichte dieses Sprichwortes wurde die Gegenthese aber immer bereits mitformuliert. ${ }^{8}$ Über Geschmack lässt sich besonders gut streiten, weil dies das Modell eines Streites unter den Bedingungen der Subjektivität ist: Diese Einsicht war bei Baumgarten das Gründungsmoment der Ästhetik als einer neuen Urteilslehre. In der Frühphase der Ästhetik war die Disziplin weder auf Autonomie noch auf Kunst bezogen. Heute gibt es das Dilemma, dass die Trennung von ethischen, ästhetischen und juridischen Autonomien nur solange funktioniert, solange man Bild- und Symbolproduktion als Synonyme zum Kunstbegriff versteht: Darin vermengt man aber gerade mediale und gesellschaftliche Phänomene. So bemerkt Frankenberg: »Im Urteil, ein Bild sei Kunst, versöhnt sich die juridische unauffällig mit der ästhetischen Bewertung und weist ibr im Recht einen Ort, genauer: einen Freiraum zu. "Dass mit einer gesellschaftstheoretischen Perspektive aber nur schwer von Versöhnung gesprochen werden kann, zeigt schon der Umstand, dass die meisten im Band behandelten Fälle von Bilderverboten gerade nicht der Sphäre der Kunst zugeordnet wurden. Bild und Kunst sind keine Synonyme. Die Zuordnung eines Bildes zur

8 Dazu: Werner Strube, Zur Geschichte des Sprichwortes »Über Geschmack lässt sich nicht streiten《. Jahrbuch für Ästhetik und allgemeine Kunstwissenschaft 30 (1985), S. 158 ff. 
Kunst ist zudem nicht einfach als juristische Subsumtionsfrage zu behandeln: Der Kunstbetrieb selbst hat bis auf wenige Ausnahmen - eine davon ist Schlingensief - eine Referenzangst entwickelt, die die Ikonophobie des Rechts spiegelt. Tatsächlich sind Kunst- und Rechtsbetrieb heute weitgehend gegeneinander abgeschottet. Die Kunst genießt wie das Recht ihre Freiräume gegenüber dem anderen. Bilder bzw. Sprachbilder und ästhetische Maßstäbe unterlaufen aber diese Autonomien. Das zeigt schon der Umstand, dass Frankenberg die Bilderverbote der Richter selber mit Bilderverboten angreift: Richter »maskieren « nach Frankenberg ihr »privates sittliches Empfinden « (18), die »Linse ihres rechtsnormativen Blickes trübt sich«(11), und beides ist ihm Zeichen für falsche juristische Entscheidungen. Masken sollen fallen, Linsen bis zur Unsichtbarkeit hin klar eingestellt werden. Diese Wendungen von Frankenberg weisen mit ihrem bildsprachlichen Bezug zu einer Ästhetik des Rechts gerade auf die unauflöslichen Schwierigkeiten einer analytischen Trennung von Ethik, Ästhetik und Recht. Frankenbergs Verteidigung juristischer Autonomie darf gerade nicht als Abwehr ästhetischer oder ethischer Maßstäbe gelesen werden. Schon die von ihm mitkonzipierte Vorlesungsreihe deutete auf den Diskursgewinn durch die Umcodierung ästhetischer bzw. ethischer in juristische Maßstäbe et vice versa. Mit der christologischen Begründung des byzantinischen Bilderstreites hat die Theologie zur Frage der Bildverbote Dissens auf höchstem Niveau erreicht. ${ }^{9}$ Aus dem Reichtum dieser Diskussion schöpft der Beitrag des Theologen Hans Joachim Höhn. Höhn vertritt die These, dass der Bilderstreit immer ein Streit um die Repräsentation von Sinn und damit um Macht ist. ${ }^{10}$ So ist es vielleicht Notwendigkeit, vielleicht $Z$ ufall, vielleicht Ironie, dass eines der ersten »Rechtsgutachten « in der Geschichte des heilig-römischen Reiches sich auf einen Bilderstreit bezieht: Die libri carolini, die um 790 von Karl d.G. bei Theo-

9 Vgl. u. a. Christian Hecht, Das Christusbild am Bronzetor. Zum byzantinischen Bilderstreit und zum theologischen Bildbegriff, in: Karl Möseneder (Hrsg.), Streit um Bilder, Berlin 1997.

10 Vgl. dazu auch: Horst Bredenkamp, Kunst als Medium sozialer Konflikte. Bilderkämpfe von der Spätantike bis zur Hussitenrevolution, Frankfurt a. M. 1975. dulf von Orléans und vermutlich bei Alkuin in Auftrag gegeben wurden, lesen sich tatsächlich nicht bloß als Stellungnahme zum Bildersturm, sondern auch als Entwurf einer »Verfassung der Repräsentation«. Als Ablauf historischer Errungenschaften markiert Höhn in seinem Beitrag die unterschiedlichen Positionen, die im Bilderstreit auftauchen: Von der orthodoxen Ansicht, dass es keine Verschiedenheit von Urbild und Abbild gibt, die nicht von einer noch größeren Bezogenheit aufeinander übertroffen werde (50), bis hin zum neuzeitlichen Konzept der Ikonographie als einem Beherrschungs- und Distanzierungskonzept gegenüber Bildern reicht dabei seine Darstellung. Es ist interessant, dass er mit seinen Ausführungen zur Logik von »Innen und Außen des Bildes « (56) auf ähnliche Überlegungen stößt wie Frankenberg. So leidet für ihn die Ausstellung »Körperwelten « mit ihrem Anspruch aufs Authentische vor allem an einem Mangel »ästhetischer Differenz « - ein Kritikpunkt, den Frankenberg zuvor mit dem Begriff des "Skandalbildes" bezeichnete. Beide verbindet die These, dass referenzlose Bilder bzw. selbstreferenzielle Bilder (38/66) jenseits des Kunstbetriebes ins Zentrum der Überlegung über Bildverbote gerückt werden sollten. Sei es, weil das Recht den Streit hier nicht an Ethik und Ästhetik zurückverweisen könne - so Frankenberg (41) -, sei es, weil der abgebildete Körper im Bild verzweckt und seiner Würde beraubt werde - so Höhn (68).

Gunnar Folke Schuppert, der den Stern Verlag bei seiner Verfassungsbeschwerde im Falle Benetton vertrat, steuert zu dem Band einen Aufsatz über den Umgang mit Mehrdeutigkeit und die Deutungshoheit bei Botschaften bei. Der Beitrag rekurriert dabei auf die medientheoretischen Untersuchungen von Klaus Röbl und Stefan Ulbrich über Bilder in der juristischen Kommunikation. Damit bietet der Beitrag das interessante Beispiel der Verknüpfung juristischer Grundlagenforschung mit einer durch die forensische Auseinandersetzung geprägten Rechtsdogmatik. Bei Schupperts Beitrag handelt es sich offensichtlich um die schriftliche Fassung seines Vortrages, und wer Schupperts Vortragskunst kennt, weiß, dass dies kaum pejorativ gemeint sein kann. Die einem Redner notwendigen Verkürzungen trüben für sich genommen den Eindruck auch nicht. Erst mit dem wiederholten Naivitätsvorwarf: gegenüber dem 
BGH fragt man sich aber, ob die Mediengeschichte des Rechts tatsächlich so zusammengefasst werden kann, wie Schuppert es tut: als »seit Aristoteles, spätestens aber seit der Renaissance « (75) schriftfixierte Kommunikation, deren typisches Beispiel heute von der Rechtsprechung des BGH geliefert werde. Es ist weniger die Zusammenfassung, die stört, als die auch bei Röhl fehlende Differenzierung zwischen Schrift und Sprache und die Annahme, dass visuelle Kommunikation anderen Sprachregeln folge als Textkommunikation. Schuppert scheint sich selbst nicht sicher, ob ihm dieser Unterschied Tatsache oder Konstrukt ist, es reicht ihm festzustellen, dass der BGH »Schwierigkeiten mit der Welt der Bilder « (77) hat. Da ist es schon überzeugender, die Argumentation des BGH als »Maskierung « zu beschreiben - wie Frankenberg es tut - denn als mediennaive Auseinandersetzung mit der Moderne.

Dies wird in der Zusammenschau mit dem folgenden Beitrag von Elisabeth Bronfen deutlich. Die Autorin versucht, einen Maßstab für angemessene Bilder zu rekonstruieren, und sie plädiert im Gegensatz zu den beteiligten Juristen durchaus auch für selbstauferlegte - Bilderverbote. Anhand der Bilder zum 11.9.2001 macht sie deutlich, dass die Geschichte der Auseinandersetzung um Bilder auch heute noch unter den rhetorischen Spannungen von ethos und pathos steht: Bilder in der Öffentlichkeit haben sich auch daran zu messen, ob sie eine angemessene Verarbeitung von Affekten erlauben. Diese Perspektive begründet Bronfen mit dem Hinweis, dass Bilder zuerst einmal das zeigen, was auch ohne sie stattfindet. $\mathrm{Zu}$ einem Image oder einem Symbol für etwas werden sie erst in der Rhetorik der Bewältigung dieses Ereignisses: Das »brennende WTC « war »zuerst einmal kein Symbol «, sondern wurde erst in der »Vergeltungsrhetorik der Bush-Regierung dazu« (107). Damit stehen Bilder in der Öffentlichkeit vor der Herausforderung, eine "psychisch und symbolisch notwendige apotropäische Geste der Entlastung « (109) bieten zu können. Aus dieser Herausforderung müssten sehr wohl Maßstäbe für die Art der Darbietung entwickelt werden. So weit wir tatsächlich von Maßstabsentwürfen im Recht entfernt sind: Die Rechtsprechung des BGH ist durch Affektkonflikte gekennzeichnet, die nicht einfach als mediennaiv abqualifiziert werden können, sondern die eben Symptom stattfindender und sich anbahnender Symbol- und Emotionskollisionen sind. Bronfens psychoanalytisch geschulter Blick eröffnet hier eine sehr eindringliche Perspektive auf neue Bildkonflikte jenseits der Liberalisierungstendenzen, die das Konfliktfeld von Sexualität und Darstellung bestimmten.

André Brodocz Beitrag bezieht sich auf ein besonderes Bildverbot, das Verbot der Kameraaufnahmen in Gerichtssälen $(\mathbb{S} 169$ GVG), das er im Hinblick auf eine gedächtnistheoretische Beschreibung der Institution des Verfassungsgerichtes beleuchtet. Der Gegenstand des Beitrages ist mit Witz gewählt: Schließlich bedient sich das Bundesverfassungsgericht in seiner Entscheidung zur Verfassungsmäßigkeit des Kameraverbotes verschiedener medienkritischer bzw. medientheoretischer Topoi und zeigte damit, dass ein Rückgriff auf aufgeklärte Bild- und Medientheorie gerade nicht zu einem Abbau von Bildverboten führen muß. So bemerkt das Bundesverfassungsgericht, dass die Massenmedien gar kein Interesse an der Realität hätten. ${ }^{11}$ Eine Einsicht, die eigentlich als sensationell vor dem Hintergrund des Konzeptes einer dienenden Kommunikationsfreiheit zu werten ist und direkt aus Luhmanns »Realität der Massenmedien «stammen könnte. Zum anderen merkt das Gericht an: »Die Aufzeichnung der mündlichen Begründung wirkt auf deren Charakter zurück. « ${ }^{12}$ und es erweist sich darin als »harter Medientheoretiker" im Sinne von Autoren wie McLuhan oder Kittler. Brodocz bewertet diese Rechtsprechung auf der Grundlage der von Aleida und Jan Assman formulierten Theorie des kulturellen Gedächtnisses - das Kameraverbot im Gerichtssaal autonomisiere insoweit ein »institutionelles Gedächtnis « des Bundesverfassungsgerichtes, und zugleich schränke die identitätsstiftende Qualität dieses Gedächtnisses die Sensibilität des Gerichtes gegenüber kulturellem Wandel ein. So bewege sich das Bundesverfassungsgericht heute mit seiner »Dämonisierung des Fernsehens « jenseits der »Praxis der herrschenden Kultur « (136).

Albrecht Obly liefert einen kritischen Beitrag zu den bildgebenden Verfahren in der Medizin. Die einschlägige Literatur zu diesem Thema war in den letzten 20 Jahren 
von einer erstaunlichen Euphorie gekennzeichnet. ${ }^{13}$ Sie spielte daher auch eine wichtige Vorreiterrolle für die geisteswissenschaftliche Diagnose eines »Iconic Turn«. Gerade in der Medizin schien sich nun ein altes bilddemiurgisches Ideal zu erfüllen: die Sichtbarmachung der Welt als deren Eroberung. Schon früh meldeten sich hieran vor allem hermeneutisch genährte Zweifel, die darauf verwiesen, dass die neuen Bilder ja auch gelesen werden müssten und die neuen Bilder an alten Problemen der Mehrdeutigkeit litten. Ohly verschärft nun diese Kritik auf der Grundlage der gegenteiligen Beobachtung, dass sich die medizinischen Bilder vor allem durch geringe Tiefenschärfe, verengtes Blickfeld und erzwungene Eindeutigkeit auszeichneten. Damit werde aber auch jede beliebige medizinische Wahrheit erzwungen. Zugleich beobachtet Ohly einen erheblichen Rückgang anderer Diagnoseformen - allem voran des Arzt-Patienten-Gespräches. Als Beitrag zum juristischen Problem des Bildverbotes liefert Ohly vor allem eine Kritik an den »monomedialen « Theorien und der Bildlichkeit, wie sie sich etwa auch bei Klaus Röhl finden.

Peter Niesens Beitrag liefert eine Darstellung der Logik des Bilderstreites unter den Bedingungen pluraler Freiheitsräume, und er knüpft damit direkt an den Beitrag von Günter Frankenberg an. Als Darstellungsmittel wählt Niesen einen Text des Popliteraten Nick Hornby. Diese Referenz ist nun nicht als Tribut an einen interdisziplinären Diskurs gewählt: Es ist das Beispiel einer reflexiven Wissenschaft, die erkennt, dass das Problem juristischer Bildverbote nicht einfach auf dem Boden juristischer Wissenschaft gelöst werden kann. Nur der Rückgriff auf einen künstlerischen Text, der als fictio normalerweise den Bilderverboten in rechtswissenschaftlichem Kontext unterfallen würde, macht deutlich, worin die Logik des Bilderstreites liegt. So sind Kunstfreiheit und Kunstzerstörung eng aufeinander bezogen: Die Kunst selber ist heute ikonoklastischer als die Rechtsprechung zu Bild-, Äußerungs- und Kunst-

13 Zur Rekapitulierung vgl. u. a. Christa Maar, Envisioning Knowledge. Die Wissensgesellschaft von Morgen, in: Christa Maar/Hans Ulrich Obrist/Ernst Pöppel (Hrsg.), Weltwissen - Wissenswelt. Das globale Netz von fext und Bild, München 2001. rechten. ${ }^{14}$ Die Verteidigung der Kunstfreiheit schränkt zugleich den Wirkungsanspruch von Bildern viel stärker ein als Zensuren. Niesen rekurriert hierzu auf den Akteur Schlingensief, der - wie oben schon angesprochen - das Recht herausfordert, indem er die Kunst herausfordert, und die Kunst herausfordert, indem er das Recht herausfordert. Ein Akteur wie Schlingensief passt in keine Erzählung über Liberalisierung und Autonomiegewinn herein. Niesen stellt die These auf, dass der Konflikt der Kunst mit dem Obszönitätsrecht als gelöst gelten kann (166). Vor dem Hintergrund der Erzählung über Schlingensief handelt es sich aber wohl eher um eine Hoffnung, als um eine These.

Robert Post schließt den Band mit einem Aufsatz über Redefreiheit, Menschenwürde und Demokratie. Der Text beschäftigt sich mit dem »belastenden und komplexen Verbältnis von Menschenwürde und Demokratie (170) und er verrät, »dass die simple Auflösung der Spannung, die in dieser komplizierten Beziebung auftreten, glücklicherweise nicht zu erwarten " ist seinem Text kommt in dem Band die Aufgabe zu, alle Fragen offen zu halten. Post beschäftigt sich mit dem Umstand, dass Autonomie und Würde in den meisten Demokratien als Gegensatz empfunden würden. Demokratie werde als Selbstbestimmungsmechanismus aufgefasst, und doch sei es gerade Aufgabe des Rechts, die Gesellschaft so einzurichten, dass die Norm der Demokratie erhalten bleibe, und es sei eine soziale Struktur, die uns erst die Norm erkennen ließe (185 f.). Der öffentliche Diskurs sei eigentlich ein Ort bösartiger Beleidigungen, eher ein Schlachtfeld denn ein »Marktplatz der Ideen« (187). So kann er im Grunde genommen schwerlich als Mittel dienen, um demokratische Legitimation und Identifikation herzustellen. So stehen wir vor dem Paradox, dass streitbare Demokratie eben immer auch selbstzerfleischende Demokratie sein kann. Posts These ist nun, dass der Umgang mit diesem Paradoxon nur möglich sei, weil das Verhältnis von Autonomie und Würde von kontingenten Besonderheiten der historischen und kulturell bedingten Urteilskraft geprägt sei und so praktisch moduliert werde. Dies sei anhand des unterschiedlichen 
Gewichts der Redefreiheit in Deutschland und Amerika erkennbar. In Deutschland sei das "geringere Gewicht « durch eine Phase kultureller Homogenität bestimmt daher sei der normative Raum des öffentlichen Diskurses von vornherein durch weniger Wetteifer geprägt (191). Weiter bezieht Post die Unterscheidung zwischen Würde und Demokratie auf eine Unterscheidung von George Herbert Mead zwischen »Me« und » $\mathrm{I}$. Eine Person zeichne sich eben durch sozialisierte "würdevolle« Struktur (»Me«) und die »demokratische« Fähigkeit aus, gesellschaftliche Aspekte ihrer selbst zu ändern oder zu überwinden (»I«) (192). Damit bleibe es immer beim Streit zwischen Würde und Demokratie.

\section{III.}

Der Band schafft es, vielfältige Bezüge zu einer konzentrierten Diskussion um Bilderverbote zu vereinen, und er bietet damit die Supervision, die ein Sammelband liefern kann. Schon die Konzeption der zugrunde liegenden Vorlesungsreihe zeichnete sich dadurch aus, dass die einzelnen Beiträge sich jeweils »diszipliniert « auf ihre Fragestellung bezogen. So sind die Untiefen des »Interdisziplinären « gemeistert. Die einzelnen Beiträge ergänzen sich zu einem Gesamtbild des "Streites um Bilder «, weil sie selbst im Streit zu einander verharren. Eine Rezension sollte nicht zum binären Code einer Kaufempfehlung (Ja/Nein) verflachen, deshalb seien hier kritisch zwei Hauptstreitlinien des Bandes herausgegriffen:

Die »Macht der Bilder « erscheint in dem Band nicht bloß als Stichwortgeber für die einzelnen Beiträge. Die meisten Autoren schließen sich der Diagnose einer besonderen Macht visueller Kommunikation an dies entspricht auch ganz weiten Kreisen der Kommunikations- und Medientheorie und der Art und Weise, wie sie in der Rechtswissenschaft rezipiert wird. ${ }^{15}$ Und ebenso wie dort, so ist auch in den vorliegenden Beiträgen nicht geklärt, ob diese Bildmacht eine Besonderheit visueller Kommunikation oder ein Aberglaube der

15 Siehe Klaus Röhl, Das Recht nach der visuellen Zeitenwende, JZ 2003, S. 339 ff. der von »subsemantischer Bildwirkung «, »Seeing is believing « und " magischer Funktion von Bildern" spricht.
Masse sein soll. Es wird ausgeblendet, dass Bildkommunikation ebenso wie Begriffskommunikation über Sprache läuft. In einem ganz grundlegenden Sinne kommunizieren Bilder eben nicht anders als geschriebene Texte: Beides wird in die Synästhesien der Kommunikationsteilnehmer übersetzt, für die Worte bereitstehen wie Begriff, Icon, Idee, Image, Anschauung, Phantasie oder Vorstellung. Die Feststellung, dass »Bilder besonders wirken« (Frankenberg), muss also relativiert werden, weil eine Schlagzeile der Bildzeitung oder ein Hymne von Hölderlin auch besonders wirken. Die Vorstellung von der Macht der Bilder ist auch nicht als Konstrukt einer bildgläubigen Masse rettbar: Damit unterstellt man anderen Leichtgläubigkeit, die man für sich selbst abweist, man verfällt also genau in die spekulative Konstruktion einer "gläubigen Menge«, die in der Rechtsprechung des BGH zu Recht kritisiert wird. Man fordert damit Bildverbote heraus, die die Konstruktion dieser Bildmacht nur verlängern. Mit den Worten Ohlys: Man stabilisiert die Hierarchie zwischen Arzt und Patient.

Die These von der »Macht der Bilder « basiert verbreitet - insbesondere in der Historiographie - auf der Vorstellung, dass Bilder ausschließlich affirmativ kommunizieren könnten: Sie könnten alles bestätigen und taugten daher nicht für einen rationalen Diskurs und seine Abwehrmöglichkeiten. ${ }^{16}$ Entweder wird, wie etwa von Röhl, die Möglichkeit einer Bildgrammatik bestritten, oder es wird behauptet, die Bildgrammatik lasse z. B. weder eine Verneinung noch ein irreales Konditional zu. Aber auch das - und das zeigt schon ein kurzer Blick in die Bildgeschichte - ist nicht richtig. Rafaels Bild vom BorgoBrand etwa zeigt einen Brand, der gerade verhindert wurde: Er zeigt etwas, das nicht stattgefunden hat, als ein erkennbar negatives Ereignis, also als irreales Konditional. Rembrandts Bild von Daniel und Cyrus vor dem Götzenbild Bel zeigt, wie man mit bildnerischen Mittel den Glauben an Bilder entkräften kann. Auch der für die moderne Kunst typische Ikonoklasmus mit bildnerischen Mitteln ist ein Beispiel für die kritischen Negationsmöglichkeiten des Bildes. Duchamps Pissoir soll schließlich weder eine formale Analogie zur Buddahfigur 
sein, noch angebetet werden - sein Readymade ist Idealtypus der aufgeklärten Enttäuschung. Bilder können ebenso wie Texte Dinge negierend auf Distanz halten - wenn man sich mit ihnen einen Begriff machen kann. Wenn sie sprachlos bleiben, kommunizieren sie auch nicht. Will man beweisen, dass es den Yeti nicht gibt, dann ist es vielleicht sinnlos, Bilder von weißen Schneeflächen zu zeigen. Man wird gar nicht wissen, ob das Photopapier entwickelt wurde, $\mathrm{ob}$ es sich um ein Bild von Robert Ryman aus dem white cube handelt oder ob der Yeti hinter der Kamera stand. Man sollte dann einfach das Bild eines bärtigen Wesens zeigen und es mit: »Ceci est Reinhold Messner unterschreiben. Womit sich der Kreis zu Magrittes Begriffsbild schließt und die Vorstellung der Macht der Bilder zusammenfällt. In dem Band zeigen die Beiträge von Elisabeth Bronfen und Hans Joachim Höhn, wie man jenseits von Machtkonstrukten über die Bedingungen von Bildverboten diskutieren kann.

Noch ein zweiter roter Faden durchzieht den Band, den man durchaus kritisch hinterfragen kann. Wiederholt taucht die Überlegung auf, dass die Geschichte des Bilderstreites in der westlichen Gesell- schaft durch Enttabuisierung- und Desensibilisierung gekennzeichnet sei. Als historische Beschreibung mag das - für eine bestimmte Epoche - stimmen. Aber eben nur dafür. Es stellt sich zudem die Frage, ob die neuen Konflikte ums Bild sich auf einer anderen Ebene abspielen, die ebenso antiliberale Tendenzen entwickeln kann. Wenn das Bild heute auch kaum mehr für Tabubrüche taugt und daher kaum mehr Zensurimpulse anstößt: Als kulturelles Symbol taugt es zumindest für »kulturelle Rahmenbrüche« - das zeigen sowohl der kontinentaleuropäische Streit ums Kopftuch als auch die Auseinandersetzungen, die sich z. B. anhand Mel Gibsons Verfilmung der Evangelien entzünden. ${ }^{17}$ Der Widerstand des Intimen scheint durch einen Widerstand des Kulturellen ersetzt zu werden - selbst ohne fundamentalistische Positionen. Noch ist nicht absehbar, welche Imagekollisionen in Zukunft Empörung hervorrufen und wann wir selber die Richter anrufen.

\section{Fabian Steinhaner}

17 Siehe hierzu die Seiten der sog. Anti Defamation League: http://www.adl.org/adl.asp. 